\title{
Treatable cardiac disease in hospitalised COPD exacerbations
}

\author{
Paul Leong ${ }^{1,2,7}$, Martin I. MacDonald ${ }^{1,2,7}$, Paul T. King ${ }^{1,2}$, Christian R. Osadnik (1) 1,3, \\ Brian S. Ko ${ }^{2,4}$, Shane A. Landry ${ }^{1,2}$, Kais Hamza ${ }^{5}$, Ahilan Kugenasan ${ }^{6}$, \\ John M. Troupis ${ }^{2,6}$ and Philip G. Bardin ${ }^{1,2}$
}

Affiliations: ${ }^{1}$ Monash Lung and Sleep, Monash Health, Clayton, Victoria, Australia. ${ }^{2}$ School of Clinical Sciences, Monash University, Clayton, Victoria, Australia. ${ }^{3}$ School of Primary and Allied Health Care, Monash University, Frankston, Victoria, Australia. ${ }^{4}$ Monash Heart, Monash Health, Clayton, Victoria, Australia. ${ }^{5}$ School of Mathematical Sciences, Monash University, Clayton, Victoria, Australia. ${ }^{6}$ Monash Imaging, Monash Health, Clayton, Victoria, Australia. ${ }^{7}$ These authors contributed equally.

Correspondence: Paul Leong, Monash Lung and Sleep, 246 Clayton Rd, Clayton, Victoria, Australia. E-mail: paul.leongamonash.edu

\section{ABSTRACT}

Introduction: Acute exacerbations of COPD (AECOPD) are accompanied by escalations in cardiac risk superimposed upon elevated baseline risk. Appropriate treatment for coronary artery disease (CAD) and heart failure with reduced ejection fraction (HFrEF) could improve outcomes. However, securing these diagnoses during AECOPD is difficult, so their true prevalence remains unknown, as does the magnitude of this treatment opportunity. We aimed to determine the prevalence of severe CAD and severe HFrEF during hospitalised AECOPD using dynamic computed tomography (CT).

Methods: A cross-sectional study of 148 patients with hospitalised AECOPD was conducted. Dynamic CT was used to identify severe CAD (Agatston score $\geqslant 400$ ) and HFrEF (left ventricular ejection fraction $\leqslant 40 \%$ and/or right ventricular ejection fraction $\leqslant 35 \%)$.

Results: Severe CAD was detected in 51 of 148 patients (35\%), left ventricular systolic dysfunction was identified in 12 cases (8\%) and right ventricular systolic dysfunction was present in 18 (12\%). Clinical history and examination did not identify severe CAD in approximately one-third of cases and missed HFrEF in two-thirds of cases. Elevated troponin and brain natriuretic peptide did not differentiate subjects with severe CAD from nonsevere CAD, nor distinguish HFrEF from normal ejection fraction. Undertreatment was common. Of those with severe CAD, only $39 \%$ were prescribed an antiplatelet agent, and $53 \%$ received a statin. Of individuals with $\mathrm{HFrEF}, 50 \%$ or less received angiotensin blockers, beta blockers or antimineralocorticoids.

Conclusion: Dynamic CT detects clinically covert CAD and HFrEF during AECOPD, identifying opportunities to improve outcomes via well-established cardiac treatments.

@ERSpublications

Severe, treatable cardiac disease is present during hospitalised \#AECOPD exacerbations, and is often clinically unsuspected. This cardiac disease can be detected with dynamic CT. Appropriate treatment could change outcomes. https://bit.ly/2Is45wO

Cite this article as: Leong P, MacDonald MI, King PT, et al. Treatable cardiac disease in hospitalised COPD exacerbations. ERJ Open Res 2021; 7: 00756-2020 [https://doi.org/10.1183/23120541.007562020].

This article has supplementary material available from openres.ersjournals.com.

This study is registered at www.anzctr.org.au with identifier number ACTRN12617001562369. Institutional ethics approval was obtained for data sharing in aggregated and deidentified formats only.

Received: 16 Oct 2020 | Accepted: 5 Nov 2020

Copyright $\odot$ ERS 2021. This article is open access and distributed under the terms of the Creative Commons Attribution Non-Commercial Licence 4.0. 


\section{Introduction}

Acute exacerbations of COPD (AECOPD) are complex, heterogenous events accompanied by a surge in cardiovascular risk and death [1-3]. Cardiac involvement, manifesting as biomarkers of myocardial dysfunction, can be detected in up to $60 \%$ of acute exacerbations [2]. When present, this heralds reduced survival $[2,3]$. Despite the frequency and importance of this signal, relationships between COPD and cardiovascular disease are imperfectly understood, particularly during AECOPD [4].

Two common, but eminently treatable cardiac diagnoses are associated with adverse outcomes at AECOPD: coronary artery disease (CAD) [5-8] and heart failure with reduced ejection fraction (HFrEF) $[9,10]$. Both cardiac disorders have well-established therapeutic pathways with clear reductions in mortality and morbidity [11-13]. They therefore represent obvious opportunities for intervention. However, their prevalence at AECOPD remains undefined, predominantly due to the limitations of routinely available diagnostic techniques.

The accurate bedside differentiation of pulmonary and cardiac diagnoses during AECOPD can be difficult. Clinical features such as dyspnoea and chest discomfort overlap and although evidence of missed ischaemic events can be found on ECGs, in the acute setting, these remain mostly nonspecific [2, 14]. Standard cardiac imaging modalities have substantive limitations during AECOPD that render them inaccurate or impractical [2, 14]. For example, transthoracic echocardiography suffers from limited acoustic windows, and cardiac magnetic resonance imaging is typically not feasible in acutely dyspnoeic individuals [2].

Computed tomography (CT) has the potential to reveal treatable cardiac disease during AECOPD. For $\mathrm{CAD}, \mathrm{CT}$ provides gold-standard quantitation of coronary artery calcium [15], an imaging marker pathognomonic of coronary atherosclerosis and atherosclerotic burden [15]. For left and right heart function, dynamic (i.e. video) CT yields extremely precise and accurate ejection fractions when judged against cardiac magnetic resonance imaging [16-20]. We combined these elements in a novel dynamic cardiopulmonary CT protocol [21] to enable rapid quantification of these key cardiac parameters in a single breath hold.

In the current work, we hypothesised that during AECOPD, major cardiac pathology would be detectable by dynamic cardiopulmonary CT, and that a significant proportion would be otherwise clinically covert, representing a substantial treatment opportunity. We aimed to assess the prevalence and treatment of two key treatable entities: CAD as determined by coronary artery calcium burden [22] and HFrEF.

\section{Methods}

\section{Patients and measurements}

A prospective observational cohort study was conducted at a tertiary metropolitan Australian hospital between December 9, 2013 and November 2, 2019 (Australian and New Zealand Clinical Trial Registry ACTRN12617001562369). In this report, we detail cross-sectional data from the index admission, reporting according to STROBE [23]. Institutional ethics approval was obtained. Some results were previously presented in abstract format [24].

Eligible participants were diagnosed with AECOPD by treating physician(s) and was subsequently verified by pulmonologists using Global Initiative for Obstructive Lung Disease [25] clinical and spirometry criteria.

Following informed consent, dynamic cardiopulmonary CT was performed as described previously [21]. Briefly, a coronary artery calcium score was obtained using the gold-standard technique of ECG gating, with results in Agatston units. This was followed by an intravenous iodinated contrast-enhanced examination simultaneously yielding left ventricular ejection fractions (LVEFs) and right ventricular ejection fractions (RVEFs) and a CT pulmonary angiogram (figure 1 and supplementary video).

Imaging was performed on a 256-slice multidetector Philips iPatent iCT (Cleveland, OH, USA) with a target dose of $4 \mathrm{mSV}$, iterative model-based reconstruction, and post-processing in Phillips Intellispace (V6, Cleveland, OH, USA) by radiographers with $>8$ years of cardiac experience. CT was performed as early as possible in the hospital admission. All subjects had estimated glomerular filtration rate $>30 \mathrm{~mL} \cdot \mathrm{min}^{-1} \cdot 1.73 \mathrm{~m}^{-2}$ and sinus rhythm, and heart rate reduction therapy was not administered. Treating physicians were blinded to CT results during hospitalisation.

Key exclusion criteria were contrast allergy and the inability to grant informed consent. Chest radiography, ECG and treatments were performed at the treating clinicians' discretion. ECG analysis was performed for study purposes by a blinded clinician with abnormal ST segments and T-wave inversion defined per the Fourth Universal Definition of Myocardial Infarction [26] (additional detail in supplementary material). 

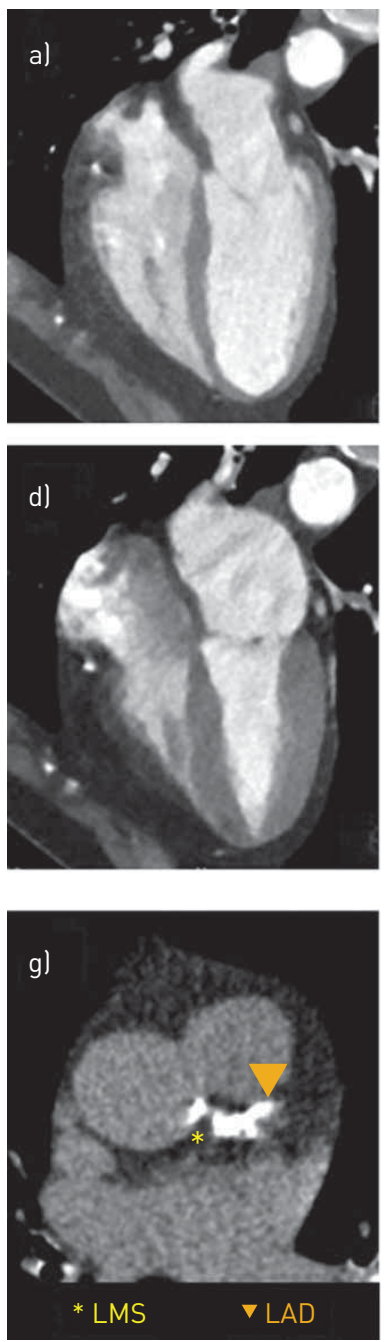
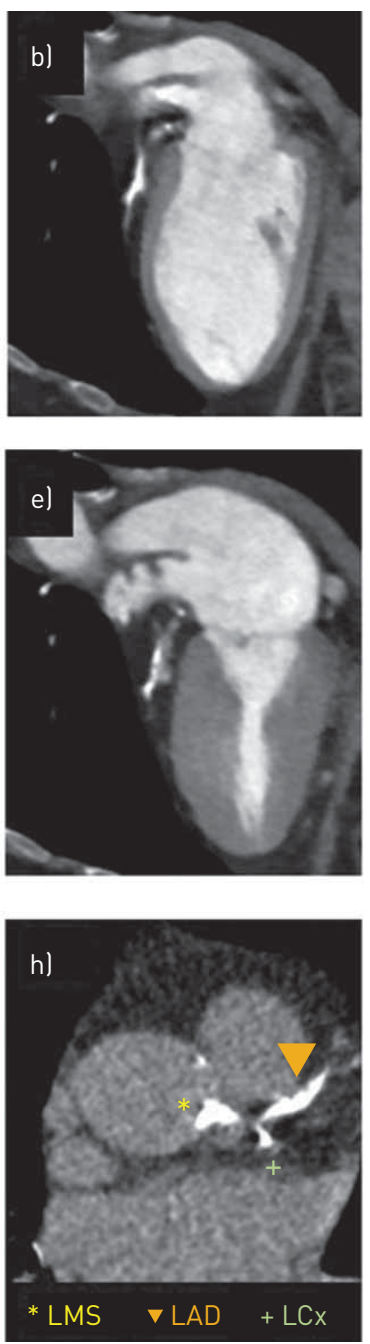
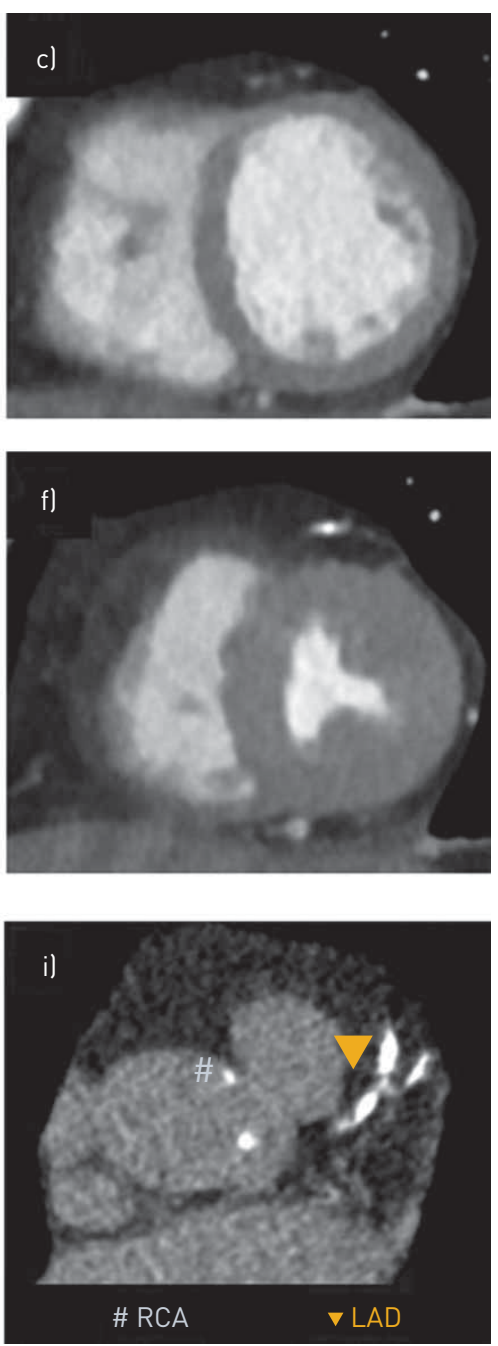

FIGURE 1 Dynamic cardiopulmonary computed tomography (CT) reveals severe but clinically undiagnosed coronary artery disease. Dynamic cardiopulmonary CT during hospitalised acute exacerbations of COPD (AECOPD) in a 77-year-old female ex-smoker with post-bronchodilator forced expiratory volume in $1 \mathrm{~s}$ of $46 \%$ predicted and no previously diagnosed cardiac disease. a-c) Representative cardiac images are shown in end-diastole and $\mathrm{d}-\mathrm{f}$ ) corresponding end-systolic images. CT revealed normal left ventricular ejection fraction $(68 \%)$ and normal right ventricular ejection fraction (55\%). g-i) Extreme coronary artery calcification was found (serial descending images), with an Agatston score of 2048. Coronary calcification was dominantly in the left main stem (LMS) and left anterior descending (LAD) arteries, with lesser disease in the left circumflex (LCX) and right coronary arteries (RCA). Troponin and B-type natriuretic peptide were not elevated. Statin and angiotensin-converting enzyme inhibitor were commenced. Following recovery from AECOPD, invasive angiography confirmed severe LMS and LAD disease requiring coronary artery bypass grafting. See also supplementary video.

At the time of study recruitment, serum samples were collected for this study. These were analysed for troponin I and B-type natriuretic peptide or N-terminal-pro B-type natriuretic peptide (hereafter, collectively BNP). Results were considered abnormal if elevated above the manufacturer's upper reference limit (age and sex-adjusted where recommended; additional detail in supplementary material). Nasopharyngeal swabs were taken for viral PCR and spontaneously expectorated sputum were submitted for bacterial culture.

\section{Outcomes and statistical analyses}

The primary outcomes were the prevalence of severe calcific CAD (Agatston score $\geqslant 400$ [27]), and severe HFrEF defined by $\mathrm{LVEF} \leqslant 40 \%$ and/or RVEF $\leqslant 35 \%$. Secondary outcomes were the frequency of cardiac treatments at the time of hospital admission, and cardiac biomarkers.

Study data were collected and managed using REDCap electronic data capture tools [28]. Analyses were performed in IBM SPSS 25 and R 3.3.3. Normally distributed data are expressed as mean \pm SD and nonparametric data as median (interquartile range). Group-wise comparisons were made with t-tests, 
Mann-Whitney U-test or Fisher's exact test as appropriate. Penalised likelihood logistic regression (logistif [6]) was used to explore predictors of 30-day readmission.

No a priori power calculations were performed and no corrections were made for multiple analyses. $\alpha$ was two-sided and set at 0.05 . Missing data were dropped from analyses.

\section{Data-sharing statement}

Institutional ethics approval was obtained for data sharing in aggregated and de-identified formats only.

\section{Results}

A total of 331 patients were screened. Exclusions were predominantly for nonsinus rhythm and severe renal dysfunction (figure 1). The final sample consisted of 148 participants (table 1 and supplementary tables 1 and 2). The median time from emergency room arrival to CT was $1.8(1.1,2.9)$ days and the mean radiation dose was $4.0 \pm 1.5 \mathrm{mSv}$. The mean heart rate at CT was $86.7 \pm 14.1$ beats. $\mathrm{min}^{-1}$ and the mean cardiac output indexed to body surface area was $3.8 \pm 0.9 \mathrm{~L} \cdot \mathrm{min}^{-1} \cdot \mathrm{m}^{-2}$ (table 2). The median coronary artery calcium score was 146.0 (28.3-821.3) Agatston units.

Patients were typical of hospitalised AECOPD cohorts $[29,30]$ with a mean age of $69.5 \pm 9.5$ years and severe airflow obstruction ( $\mathrm{FEV}_{1} 42.8 \pm 18.5 \%$ predicted); 56 (38\%) were current smokers. Viruses, most

\section{TABLE 1 Characteristics of study participants at the time of admission ( $n=148$ unless}

\section{indicated)}

Age years

Body mass index $\mathrm{kg} \cdot \mathrm{m}^{-2}$

\section{Hospitalised exacerbations in prior 12 months}

Current smoker at admission

Smoking history pack-years

Post-bronchodilator FEV /FVC $^{\#} \%$

Post-bronchodilator $\mathrm{FEV}_{1}{ }^{\#} \%$ predicted

$D_{\text {Lco }}$ \% predicted

Relevant medical history at admission

Ischaemic heart disease

Hypertension

Dyslipidaemia

Diabetes mellitus

Cerebrovascular disease

$16(11 \%)$

Heart failure

Peripheral vascular disease

Inhaled medications at admission

Long-acting muscarinic antagonist

Long-acting $\beta$-agonist

$112(76 \%)$

Inhaled corticosteroid

Domiciliary oxygen or noninvasive ventilation

\section{Cardiovascular medications at admission}

Angiotensin blocker

$\beta$-blocker

Nondihydropyridine calcium channel blocker ${ }^{+}$

Antiplatelet

Statin

Mineralocorticoid receptor antagonist

Data are presented are mean \pm SD or $n(\%) . F V_{1}$ : forced expiratory volume in $1 \mathrm{~s} ; \mathrm{FVC}$, forced vital capacity $D_{\mathrm{LCO}}$ : diffusing capacity of the lung for carbon monoxide. ${ }^{\#}: \mathrm{n}=129 ;{ }^{\uparrow}: \mathrm{n}=122{ }^{+}$: verapamil or diltiazem; $\S$ : warfarin or directly acting oral anticoagulant. 


\begin{tabular}{|c|c|}
\hline Heart rate beats $\cdot \min ^{-1}$ & $85.7 \pm 14.1$ \\
\hline \multicolumn{2}{|l|}{ Cardiac biomarkers" } \\
\hline Troponin elevated & $38 / 138(28 \%)$ \\
\hline B-type natriuretic peptide elevated & $63 / 136(46 \%)$ \\
\hline \multicolumn{2}{|c|}{ Cardiac parameters by dynamic computed tomography } \\
\hline \multicolumn{2}{|c|}{ Left ventricle } \\
\hline EDVI $\mathrm{mL} \cdot \mathrm{m}^{-2}$ & $75.1 \pm 23.0$ \\
\hline ESVI $\mathrm{mL} \cdot \mathrm{m}^{-2}$ & $30.7 \pm 22.1$ \\
\hline Ejection fraction \% & $61.6 \pm 13.7$ \\
\hline \multicolumn{2}{|l|}{ Right ventricle } \\
\hline EDVI $\mathrm{mL} \cdot \mathrm{m}^{-2}$ & $90.3 \pm 25.7$ \\
\hline $\mathrm{ESVI} \mathrm{mL} \cdot \mathrm{m}^{-2}$ & $47.2 \pm 22.7$ \\
\hline Ejection fraction \% & $48.6 \pm 10.6$ \\
\hline Left ventricular cardiac index $\mathrm{L} \cdot \mathrm{min}^{-1} \cdot \mathrm{m}^{-2}$ & $3.8 \pm 0.9$ \\
\hline \multicolumn{2}{|l|}{ Agatston coronary artery calcium score } \\
\hline 0, none & $29(20 \%)$ \\
\hline 1-99, mild & $35(24 \%)$ \\
\hline 100-399, moderate & $30(21 \%)$ \\
\hline 400-999, severe & $20(14 \%)$ \\
\hline$\geqslant 1000$, extreme & $31(21 \%)$ \\
\hline \multicolumn{2}{|c|}{$\begin{array}{l}\text { Data are presented as mean } \pm \mathrm{SD}, \mathrm{n} / \mathrm{N}(\%) \text { or } \mathrm{n}(\%) \text {. Indexed values are raw values divided by body surface } \\
\text { area. EDVI: end diastolic volume index; ESVI: end-systolic volume index. }{ }^{\#} \text { : above manufacturer's upper } \\
\text { limit of normal; }{ }^{\eta}: \mathrm{n}=145 \text { (two technical failures and one prior coronary bypass grafting). }\end{array}$} \\
\hline
\end{tabular}

commonly rhinovirus, were found in $44(30 \%)$ and bacterial pathogens were detected in $30(20 \%)$. The median eosinophil count was $0.07(0,0.15) \times 10^{9} \mathrm{~L}^{-1}$. The majority of patients (90 of $\left.148,61 \%\right)$ had been admitted to hospital for AECOPD in the previous 12 months.

\section{CAD detection}

In relation to the primary outcome, on CT, severe calcific CAD (Agatston score $\geqslant 400$ ) was found in 51 of 148 patients (35\%) (table 2 and supplementary figure 2).

Conventional clinical evaluations under-detected severe CAD (figure 2). On clinical history and examination, a diagnosis of atherosclerotic cardiovascular disease had only been made in 33 of 51 patients (61\%) who had evidence of severe coronary calcification. ECGs exhibited ST or T-wave abnormalities in 17 of $51(33 \%)$ of patients with severe CAD on CT, and 16 of $89(18 \%)$ with nonsevere CAD ( $p=0.061)$.

Troponin and BNP did not distinguish individuals with and without severe CAD on CT (supplementary table 3). Troponin was elevated in 14 of 47 (30\%) with severe CAD versus $24 / 91$ (26\%) with nonsevere CAD ( $p=0.69)$. Similarly, BNP was abnormal in 22 of $46(48 \%)$ with severe CAD versus 41 of $90(46 \%)$ under that threshold $(\mathrm{p}=0.86)$.

Six patients were diagnosed with an acute myocardial infarction by treating clinicians; all fulfilled the Fourth Universal Definition of Myocardial Infarction [26]. Two out of the six had Agatston coronary artery calcium scores $\geqslant 400$. Three patients underwent inpatient coronary angiography: one had a coronary stent placed, and another underwent emergent coronary artery bypass grafting. The remaining three patients received a clinical diagnosis of type 2 myocardial infarction and based on current standards of care, were managed noninvasively. When severe CAD was present, patients were more likely to be readmitted to hospital in the following 30 days ( 19 of 97 versus 19 of 51, $\mathrm{p}=0.029$ ).

\section{CAD treatment}

Among patients with severe CAD, at admission, only 20 of 51 (39\%) were taking an antiplatelet agent, and only 27 of $51(53 \%)$ were prescribed a statin (table 3 ).

\section{HFrEF diagnosis}

With regards to the primary outcome, using dynamic CT, LVEF $\leqslant 40 \%$ was found in 12 of $148(8 \%)$ and RVEF $\leqslant 35 \%$ was present in 18 of 148 (12\%) (supplementary tables 4 and 5). Biventricular failure was present in six individuals. 


\begin{tabular}{|c|c|c|c|c|}
\hline & Severe CAD & LVEF $\leqslant 40 \%$ & RVEF $\leqslant 35 \%$ & \\
\hline Clinical diagnosis & Percentage of patients with severe $C A D$ & Percentage of patients with LVEF $\leqslant 40 \%$ & Percentage of patients with RVEF $\leqslant 35 \%$ & $\begin{array}{l}\text { No clinical diagnosis } \\
\text { Clinical diagnosis }\end{array}$ \\
\hline $\begin{array}{l}\text { ECG or } \\
\text { chest radiography }\end{array}$ & Percentage of patients with severe CAD & Percentage of patients with LVEF $\leqslant 40 \%$ & Percentage of patients with RVEF $\leqslant 35 \%$ & \begin{tabular}{|l} 
Normal ECG \\
Abnormal ECG \\
Chest radiography \\
normal \\
Chest radiography \\
abnormal
\end{tabular} \\
\hline Troponin & Percentage of patients with severe CAD & Percentage of patients with LVEF $\leqslant 40 \%$ & Percentage of patients with RVEF $\leqslant 35 \%$ & $\begin{array}{l}\text { Troponin normal } \\
\text { Troponin elevated }\end{array}$ \\
\hline BNP & Percentage of patients with severe CAD & Percentage of patients with LVEF $\leqslant 40 \%$ & Percentage of patients with RVEF $\leqslant 35 \%$ & $\begin{array}{l}\text { BNP normal } \\
\text { BNP elevated }\end{array}$ \\
\hline
\end{tabular}

FIGURE 2 Treatable cardiac abnormalities on computed tomography (CT) are underdiagnosed by standard clinical assessments. With dynamic CT as the gold standard, percentages of patients exhibiting relevant clinical parameter are depicted in grey. The additional yield from dynamic CT is shown in black. Clinical diagnosis: clinical history and examination. CAD: coronary artery disease (Agatston score $\geqslant 400$ ); LVEF: left ventricular ejection fraction; RVEF, right ventricular ejection fraction; BNP: b-type natriuretic peptide.

Standard clinical assessments under-detected reduced LVEF (figure 2). No prior diagnosis of heart failure had been made on clinical history or examination in 8 of $12(67 \%)$ patients with low LVEF.

Chest radiographs were not predictive of reduced LVEF. In patients with reduced LVEF, only 2 of 12 $(17 \%)$ chest radiograph radiologist reports noted signs of heart failure, in comparison to 11 of $123(9 \%)$ who had normal LVEF $(\mathrm{p}=0.29)$.

TABLE 3 Cardiovascular medications at admission in patients with severe cardiac disease diagnosed by computed tomography

\section{Treatment}

Angiotensin blocker

B-blocker

Nondihydropyridine calcium channel blocker ${ }^{\#}$

Antiplatelet

Anticoagulation"

Statin

Mineralocorticoid receptor antagonist

Furosemide

\section{Coronary artery calcium score $\geqslant 400, n=51$}

$25(49 \%)$
$8(16 \%)$
$6(12 \%)$
$20(39 \%)$
$4(8 \%)$
$27(53 \%)$
$2(4 \%)$
$11(22 \%)$

Left ventricular ejection fraction $\leqslant 40 \%, n=12$

$6(50 \%)$
$2(17 \%)$
$1(8 \%)$
$6(50 \%)$
$1(17 \%)$
$8(67 \%)$
0
$1(8 \%)$

Right ventricular ejection fraction $\leqslant 35 \%, n=18$
$5(28 \%)$

$2(11 \%)$

$2(11 \%)$

$8(44 \%)$

$3(17 \%)$

$8(44 \%)$

$3(17 \%)$

$6(33 \%)$

Data are presented as $\mathrm{n}(\%) .{ }^{\#}$ : verapamil or diltiazem; ${ }^{\text {ก }}$ : warfarin or directly acting oral anticoagulant. 
Troponin was elevated in 9 of 11 (82\%) of patients with low LVEF, but was also abnormal in 29 of 127 (23\%) of those with normal LVEF. The prevalence of abnormal BNP did not differ between low LVEF and normal LVEF categories (5 of 12 (42\%) versus 58 of 124 (47\%) respectively, $\mathrm{p}=0.77$ ).

Findings were similar for RVEF $\leqslant 35 \%$. No prior diagnosis of heart failure had been made on clinical history or examination in in 12 of 18 (67\%) with low RVEF.

Chest radiographs were similarly nondiagnostic for reduced RVEF. In those with low RVEF, features of heart failure were reported in 2 of 17 (12\%) versus 11 of 129 (9\%) of those with normal RVEF ( $\mathrm{p}=0.65)$.

Troponin elevation was more frequent when RVEF was reduced, but was not limited to patients with low RVEF (10 of 15 (67\%) versus 28 of 123 (23\%), p=0.001). BNP elevation did not differentiate low RVEF and normal RVEF (5 of 17 (29\%) versus 58 of 119 (49\%), p=0.19).

Individuals with reduced LVEF had higher median coronary artery calcium scores (1532 versus 119, $\mathrm{p}<0.001)$ and lower RVEF $(34.0 \pm 12.7 \%$ versus $49.9 \pm 9.4 \%, \mathrm{p}<0.001)$ compared with those with normal LVEF (supplementary table 4). There was a tendency for higher 30-day readmission when LVEF was depressed (6 of $12(50 \%)$ versus 32 of 97 (33\%), p=0.077).

Patients with reduced RVEF had lower LVEF $(47.2 \pm 17.5$ versus $63.5 \pm 11.9, \mathrm{p}<0.001)$ and higher median coronary artery calcium scores ( 940 versus $126, \mathrm{p}=0.022$ ) (supplementary table 5 ) than those with normal RVEF. Again, 30-day readmission was numerically higher in those with low RVEF (7 of 18 (39\%) versus 31 of $130(24 \%), \mathrm{p}=0.25)$.

\section{HFrEF treatment}

At admission, in patients with low LVEF, 6 of 12 (50\%) were prescribed angiotensin blockade, however beta blocker prescription was low at 2 of 12 (17\%), and no individuals were prescribed a mineralocorticoid receptor antagonist.

Under-prescription was similarly found in patients with low RVEF. Five of 18 (28\%) received angiotensin blockade, 2 of $18(11 \%)$ received beta blockers and 3 of $18(17 \%)$ were prescribed a mineralocorticoid receptor antagonist.

Twenty-seven of $148(18 \%)$ patients received new or intensified heart failure treatment during admission. Of these, 15 of 27 (56\%) had normal biventricular function on dynamic CT.

\section{Patterns of dysfunction}

Cardiac dysfunction at AECOPD was common (figure 3). Overall, 62 of 148 (42\%) patients had one or more severe treatable cardiac abnormalities (i.e. severe CAD and/or HFrEF).

Most patients had detectable cardiac pathology: only 41 of 148 (28\%) patients did not have one or more of severe coronary artery calcification, low LVEF, low RVEF, abnormal troponin or BNP.

Overall, 44 of 135 (33\%) patients had a normal ECG, troponin and BNP. This combination made it extremely unlikely that a given individual had low left or right ventricular systolic dysfunction (which was present in 0 of 44 and 1 of 44 patients, respectively). However, this combination did not differentiate those with severe CAD from those with nonsevere CAD (13 of 44 (30\%) versus 34 of 91 (37\%), respectively, $\mathrm{p}=0.44)$.

\section{0-day readmission}

38 of $148(26 \%)$ patients were readmitted in the 30 days following hospital discharge. Three deaths occurred in this period, all of which were from respiratory causes. In univariate logistic regression, higher LVEF and $\mathrm{FEV}_{1}$ were associated with reduced odds of 30-day readmission (OR 0.965, 95\% CI 0.939-0.989, $\mathrm{p}=0.006$ and 0.969, 95\% CI $0.944-0.992, \mathrm{p}<0.001$, respectively) (supplementary table 6). Conversely, higher coronary artery calcium score percentiles were associated with increased odds of admission (OR 1.013, 95\% CI 1.001-1.025, $\mathrm{p}=0.028)$. Troponin and BNP were not associated with differences in 30-day readmission $\left(\mathrm{p}=0.293\right.$ and 0.642 , respectively). In multivariate logistic regression, only $\mathrm{FEV}_{1}$ was a significant independent predictor of 30-day readmission (OR 0.976, 95\% CI 0.949-1.0, p=0.049) (supplementary table 7).

\section{Discussion}

We report the first application of dynamic cardiopulmonary CT in a cohort of hospitalised patients with AECOPD. Severe, but treatable cardiac disease was detected in $42 \%$ of patients: severe CAD was found in about one-third of patients, and severely reduced LVEF and/or RVEF was found in approximately 10\% of cases. Standard tertiary hospital care assessments, including cardiac biomarkers performed relatively poorly to diagnose severe CAD and HFrEF. Unsurprisingly, undertreatment with disease-modifying agents 

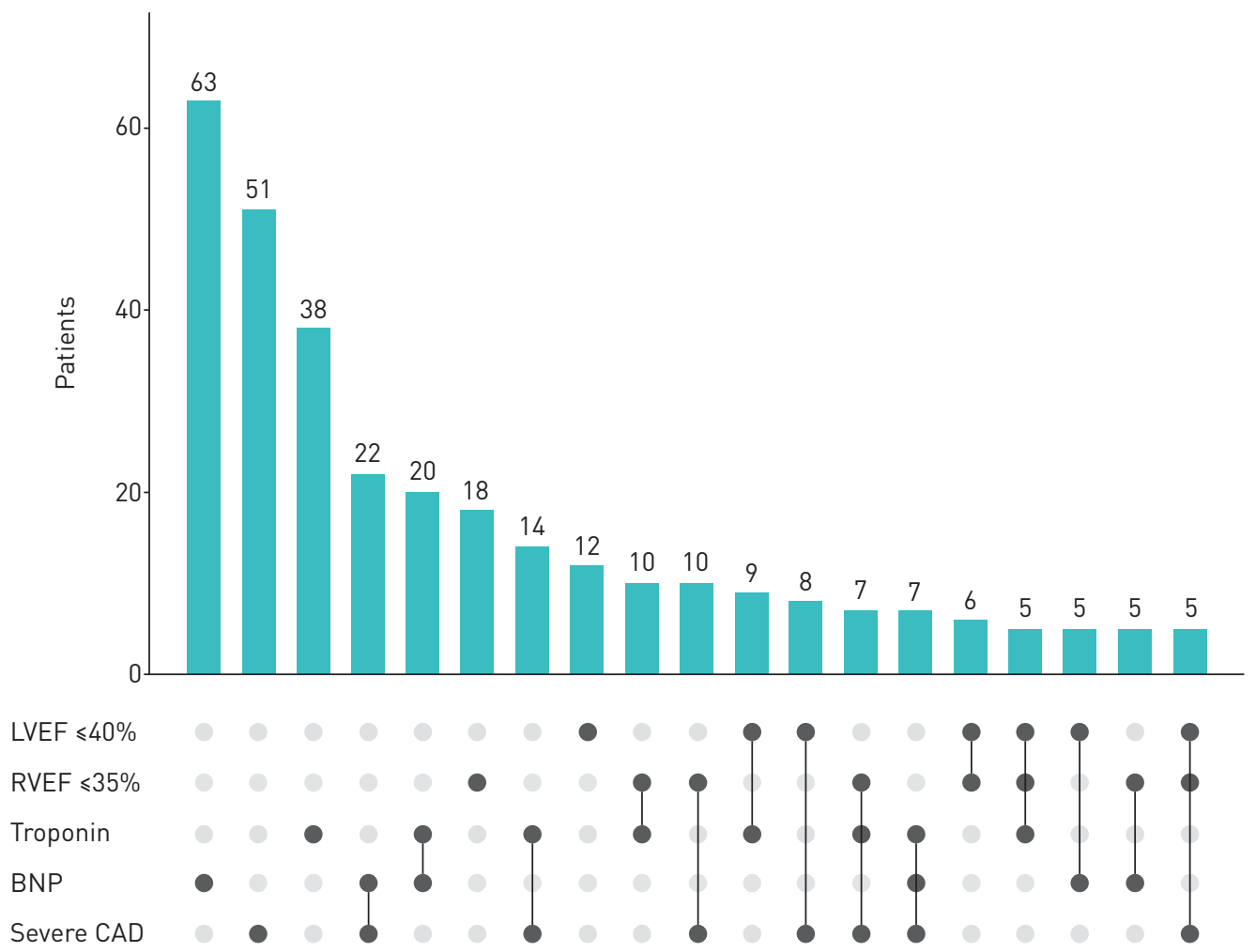

FIGURE 3 Frequency and patterns of cardiovascular involvement in 148 patients with hospitalised acute exacerbations of COPD. Frequency and patterns of associations between left ventricular ejection fraction (LVEF) $\leqslant 40 \%$, right ventricular ejection fraction (RVEF) $\leqslant 35 \%$, severely elevated coronary artery calcium score (Agatston $\geqslant 400$, severe coronary artery disease (CAD)), elevated troponin and elevated B-type natriuretic peptide (BNP). The upper bar chart shows the number of patients with the given combination of abnormalities in the lower panel. Combinations of abnormalities with a frequency of five or more individuals are shown.

was common. Taken together these findings imply that targeted disease finding in hospitalised AECOPD may aid diagnosis, treatment and prevention of future cardiac morbidity and mortality.

Hospitalised AECOPD is associated with substantial mortality and an enriched risk of subsequent major adverse cardiovascular events $[2,3]$. Hospitalisation therefore represents a favourable opportunity to diagnose and intervene in cardiac disease by implementing well-established treatment pathways with recognised treatment benefits [11-13]. The finding that $42 \%$ of patients exhibited one or more of severe CAD or HFrEF, indicates that abundant treatment opportunities exist in this population of hospitalised AECOPD. Importantly, our findings are likely to be an underestimate of cardiovascular involvement in an unselected hospitalised AECOPD population as we excluded patients with severe renal disease and those who were not in sinus rhythm.

Dynamic CT highlighted that standard clinical assessments often grossly underdiagnosed CAD and HFrEF. One-third of severe CAD and two-thirds of severe HFrEF was clinically unsuspected on standard history and examination and would have been overlooked in routine AECOPD management. ECGs were nonspecific for CAD, and chest radiographs were nonspecific for HFrEF, as were troponin and BNP. Combining standard clinical assessments could be useful as the presence of a normal ECG, troponin and BNP rendered HFrEF unlikely. This combination was not entirely reassuring as important CAD was still detected in many of these individuals and our observations require replication in other cohorts.

It is unsurprising that cardiac biomarkers were not associated with specific cardiac diagnoses since these biomarkers are sensitive but not specific for cardiovascular diagnoses. Numerous factors including exacerbation severity, effects of tachycardia, hypoxia, and chronic changes including pulmonary hypertension and diastolic dysfunction could result in biomarker elevation [2, 31]. Consequently, biomarkers cannot be relied upon to directly determine therapeutic strategies outside of defined circumstances such as dynamic troponin elevations, which might suggest acute coronary syndrome. However, biomarkers remain important sentinel markers of adverse outcomes including mortality [2]. Taken together, standard clinical assessments underdiagnosed cardiac disease and when abnormal, generally lacked the diagnostic accuracy required to institute disease-specific treatments. 
The current studies show that $35 \%$ of patients had severe CAD, mirroring other cohorts including ECLIPSE, COPDGene and the Danish Lung Cancer Screening Trial [7, 8, 32]. In populations with COPD, $\mathrm{CAD}$ is associated with adverse outcomes, including higher dyspnoea, lower exercise capacity and greater mortality [7, 8, 32]. Further, the incidence of acute myocardial infarction increases at AECOPD [3] beyond a baseline of elevated risk [33]. In aggregate, diagnosing CAD during AECOPD represents an opportunity for cardiac risk mitigation not only during that episode, but also towards improved longer-term outcomes. This notion is supported by data from COPD populations suggesting that antiplatelet and lipid-lowering treatments may reduce dyspnoea, mortality and COPD exacerbations [1,4]. A case-finding approach for $\mathrm{CAD}$, such as that using CT, could define individuals with CAD and its severity, allowing targeted cardiac risk reduction.

HFrEF was less common in our cohort at about $10 \%$ prevalence, but this group may be of disproportionate clinical prominence since coexistent heart failure and COPD is associated with an increased frequency of hospitalisation $[2,4]$ and incipient heart failure may simulate AECOPD or even precipitate it $[2,4,14]$. Diagnosis and treatment of HFrEF appeared clinically difficult in our study. Two-thirds of those patients with HFrEF on dynamic CT related no clinical history or examination evidence of this diagnosis. Conversely, about half of patients with normal biventricular function received treatment for presumed heart failure. Identifying COPD patients with clear indications for HFrEF treatment such as angiotensin blockade and beta blockers would enhance the risk-benefit profile of these agents, as broader prescription may lead to adverse effects [34]. Finally, when HFrEF was detected, a higher burden of CAD was present, suggesting that CAD warrants consideration when HFrEF is detected.

Current AECOPD treatment paradigms [25] are dominantly aimed at pulmonary, rather than cardiac parameters, despite COPD populations demonstrating a 2.5-fold increased risk of cardiac disease [33]. Paradoxically, COPD management recommendations emphasise a thorough 'precision medicine' approach, in which characteristics are defined and treated [35]. We found that some form of cardiac involvement at AECOPD was extremely common, with only $28 \%$ of patients not exhibiting one or more of severe CAD, HFrEF or abnormal cardiac biomarkers. Without knowledge of influential cardiac diagnoses, a precision medicine strategy is difficult to fully implement, and treatable cardiac diseases could be routinely overlooked. Overall, accurately diagnosing and treating cardiac comorbidities at an opportune occasion such as AECOPD could not only alter mortality and morbidity $[1,4,11-13]$ but could also ensure tailored pulmonary treatments that will minimise adverse cardiac effects. Our data demonstrated, however, that $\mathrm{FEV}_{1}$ was the parameter most strongly associated with 30-day readmission, implying that optimising airway management may be the correct approach to preventing short-term readmission for many patients.

The current study has caveats. Severely unwell patients, including those with arrhythmias and with poor renal function, were excluded from the study. It is possible that results may not apply outside the study population of hospitalised AECOPD. However, findings are broadly consistent with previous global reports of under-diagnosis and undertreatment of coronary disease and systolic heart failure in COPD populations $[1,7,36-38]$. For example, the quantitative coronary calcium burden in our patients was comparable with that in the ECLIPSE cohort [7], who also only received statins about one-quarter of the time; beta blocker under-prescription for HFrEF is also commonly reported [1, 37, 39].

Diastolic dysfunction, and the clinical entity of heart failure with preserved ejection fraction are key comorbidities in patients with COPD that may explain cardiac biomarker elevation in some patients. However, methodologies to ascertain and quantify diastolic dysfunction on dynamic CT are not yet well established. Additionally, there is currently limited evidence that specific pharmacotherapies alter the course of heart failure with preserved ejection fraction, in contradistinction to HFrEF and CAD. Examining diastolic dysfunction therefore merits further study but was outside the scope of this work.

A limitation of this work is that multiple assays were used for both troponin and BNP. Because there are no validated ways to interchange values between assays, we were unable to draw inferences based on absolute biomarker levels. In addition, blood lipids were not measured and so could not be used to apply standard cardiovascular risk estimators.

A key strength is the use of dynamic CT to accurately assesses important cardiac parameters in a single, rapid imaging session requiring only one breath hold [21]. Coronary calcium was quantified using the gold-standard technique of prospective ECG-gated CT [15, 22]. For left and right ventricular parameters, dynamic CT is extremely precise and accurate, exhibiting near-perfect correlation (Pearson r 0.9 to 0.99 ) with the gold-standard of magnetic resonance imaging $[16,17,19,20]$. CT however does require radiation exposure, adequate renal function and cannot directly measure pressures or vascular resistance.

The detection of coronary artery calcium reflects late-stage subclinical coronary atherosclerosis, and scores are proportionate to the area and volume of plaque $[15,22,40]$. Although we used the term severe CAD 
to refer to Agatston scores $\geqslant 400$ based on precedent [27], and worse outcomes when scores $\geqslant 400[8,41]$, it is a limitation that we did not conclusively demonstrate that these were associated with specific disease entities such as ischaemic heart disease.

During AECOPD, dynamic CT affords substantial pragmatic advantages in comparison with other cardiac imaging modalities. In many patients with COPD, transthoracic echocardiography parameters cannot even be obtained due to hyperinflation and poor acoustic windows [2, 21]. In addition to overcoming this practical disadvantage, dynamic CT is more accurate than echocardiography [16]. Magnetic resonance imaging requires prolonged acquisitions in a claustrophobic environment, an arduous and potentially dangerous undertaking during acute breathlessness. Neither echocardiography nor magnetic resonance imaging can quantitate CAD. Conversely, dynamic CT was practicable and well tolerated during hospitalised AECOPD, revealing two significant, treatable cardiac diseases.

Future studies could answer whether the HFrEF abnormalities observed are persistent or transient, and clarify associations between outcomes and other CT-derived measurements. Key unresolved questions are identifying a subpopulation who would benefit from an invasive angiographic approach, and whether routine implementation of a CT-based case-finding strategy would be cost-efficient and improve long-term outcomes.

In conclusion, detection of cardiac disease employing dynamic cardiopulmonary CT is feasible during AECOPD with approximately $40 \%$ of patients having severe, but treatable cardiac disease. The ability to accurately delineate cardiac pathologies has potential to guide treatment and improve prognosis in people with COPD.

Acknowledgements: The authors thank Anne Tran and Gayan Kathriachchige (both Monash Lung and Sleep) for their important contributions to this work.

Author contributions: Conception, design: P. Leong, M.I. MacDonald, P. King, C.R. Osadnik, B.S. Ko, A. Kugenasan, J.M. Troupis and P.G. Bardin; acquisition and analysis: P. Leong, M.I. MacDonald, B.S. Ko, S.A. Landry, K. Hamza, A. Kugenasan and J.M. Troupis; interpretation: P. Leong, M.I. MacDonald, P. King, C.R. Osadnik, B.S. Ko, J.M. Troupis and P.G. Bardin; first draft: P. Leong. All authors participated in revising the work, agreed to submit this version for publication, and agree to be accountable for all aspects of the work.

Conflict of interest: P. Leong has nothing to disclose. M.I. MacDonald has nothing to disclose. P. King has nothing to disclose. C.R. Osadnik has nothing to disclose. B.S. Ko reports personal fees from Canon Medical during the conduct of the study. S.A. Landry has nothing to disclose. K. Hamza has nothing to disclose. A. Kugenasan has nothing to disclose. J.M. Troupis has nothing to disclose. P.G. Bardin has nothing to disclose.

Support statement: P. Leong is supported by the Australian National Health and Medical Research Council Postgraduate Scholarship, the Royal Australasian College of Physicians Dixon Award, and the Monash Lung and Sleep Institute. C.R. Osadnik was the recipient of a Lung Foundation Australia COPD Research Fellowship and is the recipient of a Rebecca L. Cooper Medical Research Foundation Project Grant, unrelated to the present work. Views expressed are those of the authors and may not represent those of the funding bodies. The funders had no role in study design, data collection and analysis, decision to publish or preparation of the manuscript. Funding information for this article has been deposited with the Crossref Funder Registry.

\section{References}

1 Morgan AD, Zakeri R, Quint JK. Defining the relationship between COPD and CVD: what are the implications for clinical practice? Ther Adv Respir Dis 2018; 12: 175346581775052.

2 MacDonald MI, Shafuddin E, King PT, et al. Cardiac dysfunction during exacerbations of chronic obstructive pulmonary disease. Lancet Respir Med 2016; 4: 138-148.

3 Wang M, Lin EP-Y, Huang L-C, et al. Mortality of cardiovascular events in COPD patients with preceding hospitalized acute exacerbation. Chest 2020; 158: 973-985. .

4 Rabe KF, Hurst JR, Suissa S. Cardiovascular disease and COPD: dangerous liaisons? Eur Respir Rev 2018; 27: 180057.

5 Patel ARC, Donaldson GC, Mackay AJ, et al. The impact of ischemic heart disease on symptoms, health status, and exacerbations in patients with COPD. Chest 2012; 141: 851-857.

6 McGarvey LP, John M, Anderson JA, et al. Ascertainment of cause-specific mortality in COPD: operations of the TORCH Clinical Endpoint Committee. Thorax 2007; 62: 411-415.

7 Williams MC, Murchison JT, Edwards LD, et al. Coronary artery calcification is increased in patients with COPD and associated with increased morbidity and mortality. Thorax 2014; 69: 718-723.

8 Bhatt SP, Kazerooni EA, Newell JD, et al. Visual estimate of coronary artery calcium predicts cardiovascular disease in COPD. Chest 2018; 154: 579-587.

9 Fuso L, Incalzi RA, Pistelli R, et al. Predicting mortality of patients hospitalized for acutely exacerbated chronic obstructive pulmonary disease. Am J Med 1995; 98: 272-277.

10 Boudestein LCM, Rutten FH, Cramer MJ, et al. The impact of concurrent heart failure on prognosis in patients with chronic obstructive pulmonary disease. Eur J Heart Fail 2009; 11: 1182-1188.

11 Arnett DK, Blumenthal RS, Albert MA, et al. 2019 ACC/AHA guideline on the primary prevention of cardiovascular disease: a report of the American College of Cardiology/American Heart Association Task Force on clinical practice guidelines. Circulation 2019; 140: e596-e646. 
12 Grundy SM, Stone NJ, Bailey AL, et al. 2018 AHA/ACC/AACVPR/AAPA/ABC/ACPM/ADA/AGS/APhA/ASPC/ NLA/PCNA guideline on the management of blood cholesterol: a report of the American College of Cardiology/ American Heart Association Task Force on clinical practice guidelines. J Am Coll Cardiol 2019; 73: e285-e350.

13 Yancy CW, Januzzi JL, Allen LA, et al. 2017 ACC expert consensus decision pathway for optimization of heart failure treatment: answers to 10 pivotal issues about heart failure with reduced ejection fraction: a report of the American College of Cardiology Task Force on expert consensus decision pathways. J Am Coll Cardiol 2018; 71: 201-230.

14 Leong P, Macdonald MI, Ko BS, et al. Coexisting chronic obstructive pulmonary disease and cardiovascular disease in clinical practice: a diagnostic and therapeutic challenge. Med J Aust 2019; 210: 417-423.

15 Hecht H, Blaha MJ, Berman DS, et al. Clinical indications for coronary artery calcium scoring in asymptomatic patients: expert consensus statement from the Society of Cardiovascular Computed Tomography. J Cardiovasc Comput Tomogr 2017; 11: 157-168.

16 Greupner J, Zimmermann E, Grohmann A, et al. Head-to-head comparison of left ventricular function assessment with 64-row computed tomography, biplane left cineventriculography, and both 2- and 3-dimensional transthoracic echocardiography. J Am Coll Cardiol 2012; 59: 1897-1907.

17 Maffei E, Messalli G, Martini C, et al. Left and right ventricle assessment with cardiac CT: validation study vs. cardiac MR. Eur Radiol 2012; 22: 1041-1049.

18 Sarwar A, Shapiro MD, Nasir K, et al. Evaluating global and regional left ventricular function in patients with reperfused acute myocardial infarction by 64-slice multidetector CT: A comparison to magnetic resonance imaging. J Cardiovasc Comput Tomogr 2009; 3: 170-177.

19 Raman SV, Shah M, McCarthy B, et al. Multi-detector row cardiac computed tomography accurately quantifies right and left ventricular size and function compared with cardiac magnetic resonance. Am Heart J 2006; 151: 736-744

20 Wu Y-W, Tadamura E, Kanao S, et al. Left ventricular functional analysis using 64-slice multidetector row computed tomography: comparison with left ventriculography and cardiovascular magnetic resonance. Cardiology 2008; 109: 135-142.

21 Leong P, MacDonald MI, Ko BS, et al. Single-breath comprehensive cardiopulmonary assessment utilizing computerized tomography. Respirology 2019; 24: 1026-1029.

22 Greenland P, Blaha MJ, Budoff MJ, et al. Coronary calcium score and cardiovascular risk. J Am Coll Cardiol 2018; 72: $434-447$

23 von Elm E, Altman DG, Egger M, et al. The strengthening the reporting of observational studies in epidemiology (STROBE) statement: guidelines for reporting observational studies. Ann Intern Med 2007; 147: 573-577.

24 MacDonald MI, Wong A-M, King P, et al. Occult Cardiac Disease Can Be Identified by Dynamic 256-Slice CT During Chronic Obstructive Pulmonary Disease Exacerbation. Am J Respir Crit Care Med 2016; 193: A2982.

25 Global Initiative for Chronic Obstructive Lung Disease (GOLD). Global Strategy for the Diagnosis, Management and Prevention of COPD. GOLD, 2020.

26 Thygesen K, Alpert JS, Jaffe AS, et al. Fourth universal definition of myocardial infarction (2018). J Am Coll Cardiol 2018; 72: 2231-2264.

27 Raggi P, Callister TQ, Cooil B, et al. Identification of patients at increased risk of first unheralded acute myocardial infarction by electron-beam computed tomography. Circulation 2000; 101: 850-855.

28 Harris PA, Taylor R, Thielke R, et al. Research electronic data capture (REDCap)-A metadata-driven methodology and workflow process for providing translational research informatics support. J Biomed Inform 2009; 42: 377-381.

29 Wark PAB, Tooze $\mathrm{M}$, Powell $\mathrm{H}$, et al. Viral and bacterial infection in acute asthma and chronic obstructive pulmonary disease increases the risk of readmission. Respirology 2013; 18: 996-1002.

30 Ko FW, Chan KP, Hui DS, et al. Acute exacerbation of COPD. Respirology 2016; 21: 1152-1165.

31 Anderson WJ, Lipworth BJ, Rekhraj S, et al. Left ventricular hypertrophy in COPD without hypoxemia. Chest 2013; 143: 91-97.

32 Rasmussen T, Køber L, Abdulla J, et al. Coronary artery calcification detected in lung cancer screening predicts cardiovascular death. Scand Cardiovasc J 2015; 49: 159-167.

33 Chen W, Thomas J, Sadatsafavi M, et al. Risk of cardiovascular comorbidity in patients with chronic obstructive pulmonary disease: a systematic review and meta-analysis. Lancet Respir Med 2015; 3: 631-639.

34 Dransfield MT, Voelker H, Bhatt SP, et al. Metoprolol for the prevention of acute exacerbations of COPD. $N$ Engl J Med 2019; 381: 2304-2314.

35 Agusti A, Bel E, Thomas M, et al. Treatable traits: toward precision medicine of chronic airway diseases. Eur Respir J 2016; 47: 410-419.

36 Brekke PH, Omland T, Smith P, et al. Underdiagnosis of myocardial infarction in COPD - cardiac infarction injury score (CIIS) in patients hospitalised for COPD exacerbation. Respir Med 2008; 102: 1243-1247.

37 Quint JK, Herrett E, Bhaskaran K, et al. Effect of beta blockers on mortality after myocardial infarction in adults with COPD: population-based cohort study of UK electronic healthcare records. BMJ 2013; 347: f6650-f6650.

38 Pizarro C, Herweg-Steffens N, Buchenroth $\mathrm{M}$, et al. Invasive coronary angiography in patients with acute exacerbated COPD and elevated plasma troponin. Int J Chron Obstruct Pulmon Dis 2016; 11: 2081-2089.

39 de Miguel Díez J, Morgan JC, García RJ. The association between COPD and heart failure risk: a review. Int J Chron Obstruct Pulmon Dis 2013; 8: 305-312.

40 Piepoli MF, Hoes AW, Agewall S, et al. 2016 European Guidelines on cardiovascular disease prevention in clinical practice: the Sixth Joint Task Force of the European Society of Cardiology and Other Societies on Cardiovascular Disease Prevention in Clinical Practice (constituted by representatives of 10 societies and by invited experts): developed with the special contribution of the European Association for Cardiovascular Prevention \& Rehabilitation (EACPR). Eur J Prev Cardiol 2016; 23: NP1-NP96.

41 Arad Y, Goodman KJ, Roth M, et al. Coronary calcification, coronary disease risk factors, C-reactive protein, and atherosclerotic cardiovascular disease events: the St. Francis Heart Study. J Am Coll Cardiol 2005; 46: 158-165. 